\title{
Stereoscopic Volume Rendering of Medical Imaging Data for the General Public
}

Franz A Fellner ${ }^{1,2^{*}}$, Florian Berger ${ }^{3}$, Christine Fellner ${ }^{1},{\text { Christoph } \text { Kremer }^{3} \text {, Horst Hörtner }}^{3}$ and Gerfried Stocker ${ }^{3}$

${ }^{1}$ Institute of Radiology, Kepler University Clinic, Medical Faculty of the Johannes Kepler University, Linz, Austria

${ }^{2}$ Medical Faculty of the Friedrich-Alexander-University Erlangen-Nürnberg, Erlangen, Germany

${ }^{3}$ Ars Electronica, Linz, Austria

\section{Introduction}

The Ars Electronica Center (AEC) is known as the Museum of the Future. As such, one of its specialties is exhibiting multifarious hybrids and blends of various artistic genres, scientific fields and technological developments and elaborating on them. Biotechnology and genetic engineering, neurology, robotics, prosthetics and media art meet here on an equal footing as they constitute experimental arrays for testing how we might be coexisting and communicating with our physical surroundings as well as other human beings in the not-too-distant future, and indicating what these changes will mean for us and our society.

Plus, the Ars Electronica Center offers its visitors something that, in the form it assumes here, is absolutely unique worldwide: a projection space in which an audience of 150 can behold $16 \times 9$-meter images on a forward wall surface as well as $16 \times 9$-meter images on the floor in front of them. These visuals require eight projectors-four for the wall, four for the floor. This makes laser tracking and 3-D animation available to the general public (Figure 1).

\section{Medicine and the natural sciences for the general public}

Approximately eight years ago, the authors came up with the idea of also presenting medical subjects to the general public here-for example, modern aspects of medicine (anatomy of the human brain, possibilities of examining the brain using MR imaging) and especially of radiology (e.g. functional MR imaging, tractography), the principles upon which CT and MR are based and the sequences of steps these examinations entail (Figure 2). These presentations were originally conceived as one-shot-deal events, but their success exceeded the organizers' most optimistic expectation. The upshot: the development of a series of continuing education events now staged on a regular basis at the AEC.

\section{Stereoscopy in deep space}

Deep Space is not only a setting for high-resolution projections displayed on dual $16 \times 9$-meter surfaces on the wall and floor. It also features technology to present stereoscopic 3-D images, 3-D films and interactive 3-D real-time graphics. Here, visitors outfitted with 3-D shutter glasses can enjoy a truly impressive three-dimensional experience.

A year ago, this possibility inspired a fascinating idea: presenting medical imaging examinations (CT and MR data sets) in 3-D here. But the first problem was to make these data sets 3D-enabled. One of us-F.B., an Ars Electronica FutureLab staffer-finally solved this problem by writing a volume rendering program that was capable of directly importing regular DICOM data (exactly like those we generate in radiology), processing them and presenting them without having to manually process them in advance (Figure 3).

For stereoscopic volume rendering, three different methods, all based on ray tracing (no meshes involved), were used to display different aspects of the imaging data sets (Figure 4):

1) Density Threshold (detect surface by jump of density over a certain threshold)

2) Gradient Weighting (detect surface normal by big gradients in data set)

3) Density Filter (filter dataset for a specific density within a certain density window)

The methods were implemented without any reference to existing methods. We assume that similar principles are used by other existing approaches.

Spatial resolution of the applied CT and MR imaging data ranges between (depending on body area and imaging method) $0.5 \times 0.5 \times 0.6$ $\mathrm{mm}^{3}$ and $0.8 \times 0.8 \times 0.8 \mathrm{~mm}^{3}(\mathrm{MR})$ respectively $0.4 \times 0.4 \times 1.0 \mathrm{~mm}^{3}$ and $0.6 \times 0.6 \times 1.0 \mathrm{~mm}^{3}(\mathrm{CT})$.

For presentation a workstation is installed in Deep Space prior to an event and manned during the presentation by F.B. or another FutureLab staffer. Thus, what takes place here is live volume rendering of the image data, which makes this experience even more enthralling for the audience.

Modification of the density values (CT) or intensity values (MR) makes it possible to depict various strata of the human body. Usually, we begin with a setting for the body surface or the surface of the part of the body that is being examined.

During a smoothly adjustable online rotation, the patient is rotated $360^{\circ}$ so the audience can see how the patient was lying on the CT table (Figure 3).

Then, we proceed successively deeper into the interior of the body. Along the way, we point out the various anatomical structures of the human body (bones, thorax, abdomen, different abdominal organs, and vessels) (Figures 4 and 5).

The data sets can also be smoothly enlarged, shifted and edited online, which makes it possible to focus on various different parts of the body and the organs in them. It's also possible to select from among several modes of depiction and to directly fine-tune their density or

*Corresponding author: Franz A Fellner, Institute of Radiology, Kepler University, Clinic Krankenhaus, Str. 9, 4020 Linz, Austria,Tel: 43732 24680; E-mail: franz.fellner@akh.linz.at

Received October 12, 2015; Accepted January 07, 2016; Published January 14 2016

Citation: Fellner FA, Berger F, Fellner C, Kremer C, Hörtner H, et al. (2016) Stereoscopic Volume Rendering of Medical Imaging Data for the General Public. J Health Med Informat 7: 213. doi:10.4172/2157-7420.1000213

Copyright: (c) 2016 Fellner FA, et al. This is an open-access article distributed under the terms of the Creative Commons Attribution License, which permits unrestricted use, distribution, and reproduction in any medium, provided the original author and source are credited. 
Citation: Fellner FA, Berger F, Fellner C, Kremer C, Hörtner H, et al. (2016) Stereoscopic Volume Rendering of Medical Imaging Data for the General Public. J Health Med Informat 7: 213. doi:10.4172/2157-7420.1000213

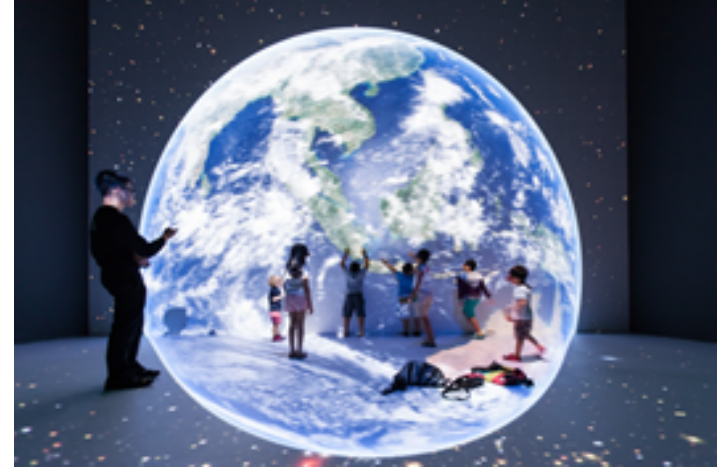

Figure 1: A typical deep space presentation (Theme: Planet Earth); outfitted with 3-D shutter glasses, the audience sees the images stereoscopically.



Figure 2: Deep space presentation on the subject of imaging procedures used in medicine and anatomy.

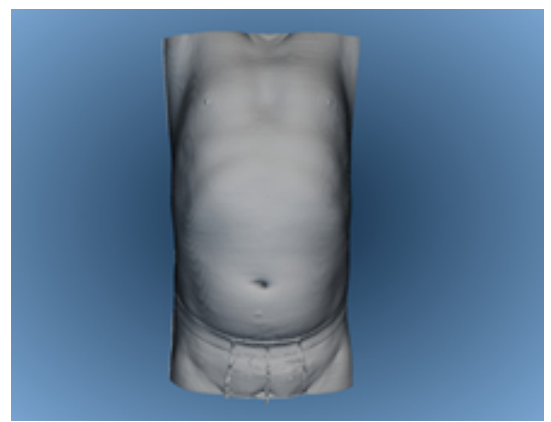

Figure 3: Volume rendering of a full-body CT data set with reconstruction of the surface of the patient's body.

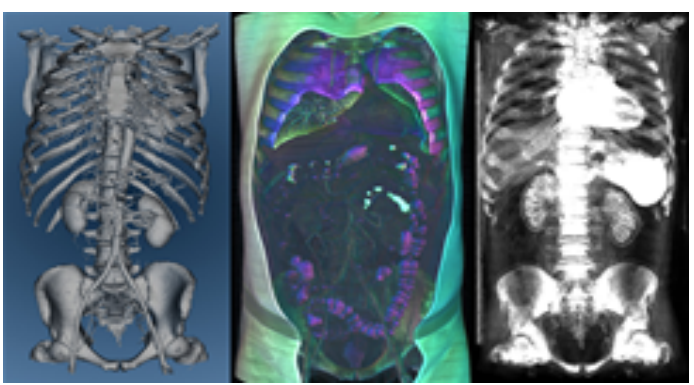

Figure 4: Full-body CT data sets as in Figures 3 and 4: surface reconstruction via density threshold value (left); surface reconstruction via density gradient weighting (middle); detection of regions with a particular density via density filter (right). surface threshold values at will during the presentation so that a wide array of structures (e.g. bones, blood vessels and parenchyma structures such as bronchia) can be optimally depicted (Figure 6).

At present, there is still hardly any literature on the subject of stereoscopic volume rendering. A Medline search using the search terms "stereoscopic volume rendering" yields a mere 39 publications; entering "volume rendering" yields 2,358 works.

The 39 works on this subject deal primarily with the advantages of three-dimensional viewing of imaging examinations for attending surgeons-for example, in the case of a pleural mesothelioma [1] for the thoracic surgeon, a neurosurgeon's preparations for an operation on an intracranial aneurysm [2], or for neurosurgery in general $[3,4]$. Further publications report applications in gynecology [5] and otolaryngology [6].

Our research in the literature has yet to yield descriptions of the situation as we have described it here.

The reason why the three-dimensional images from Deep Space appear blurred here is that the camera-in contrast to a human spectator-sees both images simultaneously and records them overlapping, whereas spectators in Deep Space wear special glasses that separate the image for the left eye from the image for the right eye. This is what produces the impression of depth, and also makes the images crystal-clear (See the projection in Figure 2, which is not a three-dimensional image).

\section{Previously presented investigations}

The following investigations have already been presented at various continuing education offerings: CT whole body, as described above; also, contrast-enhanced MR angiography of neck arteries (Figure 7), and non-contrast 3D time-of-flight (TOF) MR angiography of intracranial arteries.

\section{Special features}

At a very early stage-in the wake of the first presentation-we became aware of another problem. It was impossible to use a laser pointer to show the various structures mentioned above because, for one thing, the point of light was too weak, and, for another, because a spatial shift of the point occurred when aimed at an area outside of the center (from the demonstrator's point of view). F.B. responded to this issue by writing a program that enables the demonstrator to use a commercially available tablet to exercise online control over a virtual three-dimensional arrow (Figure 8) that can be manipulated not only two-dimensionally on the $\mathrm{x}$-axis and $\mathrm{y}$-axis but also in the third spatial dimension, the $\mathrm{z}$-axis of depth.

In the next developmental phase, F.B. implemented a labeling option that can be used to mark and label any structures the demonstrator desires (Figure 9). When the demonstrator undertakes a zoom, shift or rotation online, the markings and labels move along with the data set so as to remain spatially correct.

\section{Future: Implications for the Medical Domain?}

Until now the focus is on the topic to provide stereoscopic volume rendering of medical imaging data to the general public. For this purpose, only CT and MR data sets with normal findings are presented.

A current issue of research in medicine is the value of stereoscopical assessment of volume-rendered medical imaging data in comparison to traditional monoscopic viewing. A recent paper of Stewart et al 


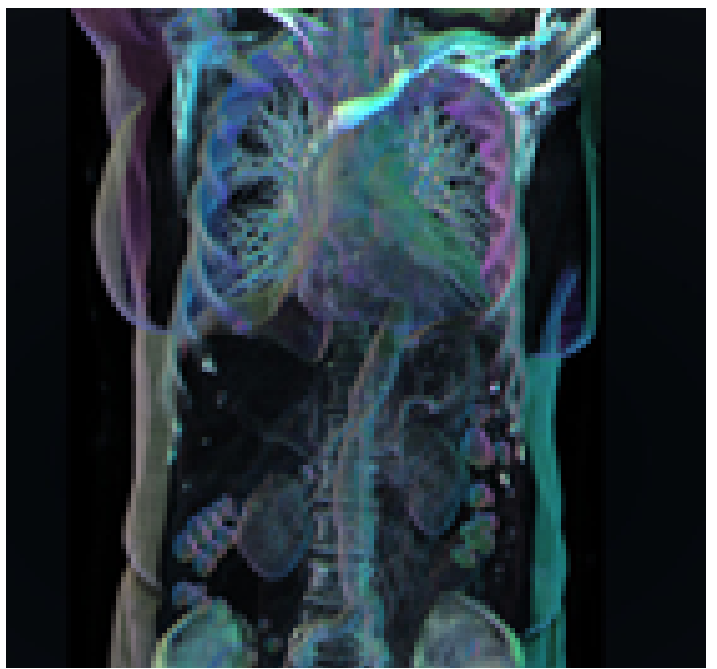

Figure 5: Whole body CT: stereoscopic visualization on a $16 \times 9$-meter projection surface in Deep Space provided by a DICOM data volume rendering program developed in-house by the Ars Electronica FutureLab's F.B.



Figure 6: Zoom and shift to the thorax region; the contrasts and threshold values are set so as to make the respiratory passages (trachea and bronchia) optimally visible.

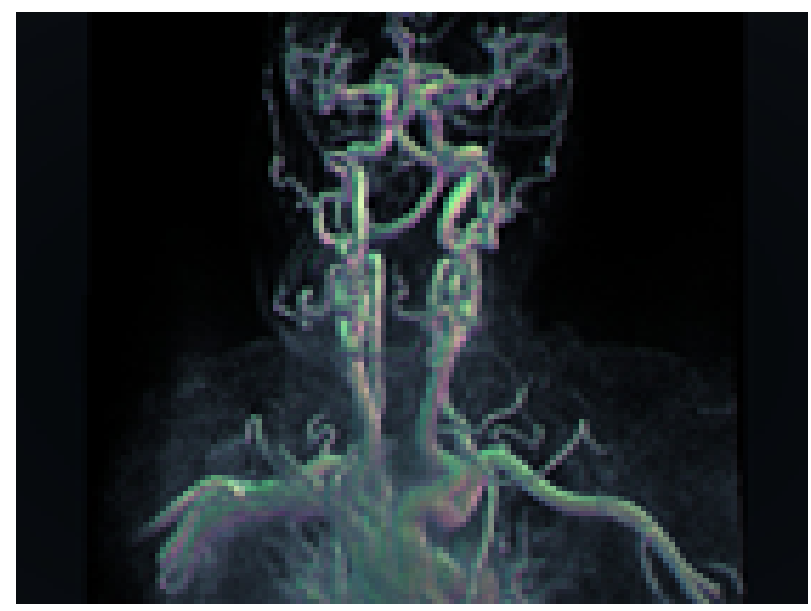

Figure 7: Stereoscopic volume rendering in Deep space of a CE MRA of the neck arteries.
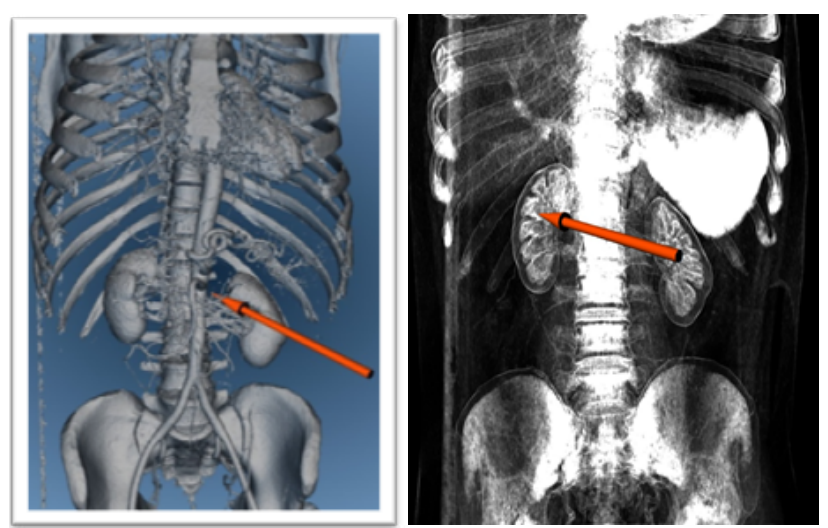

Figure 8: Examples of the use of a three-dimensional arrow pointer: aorta (left); right kidney (right).

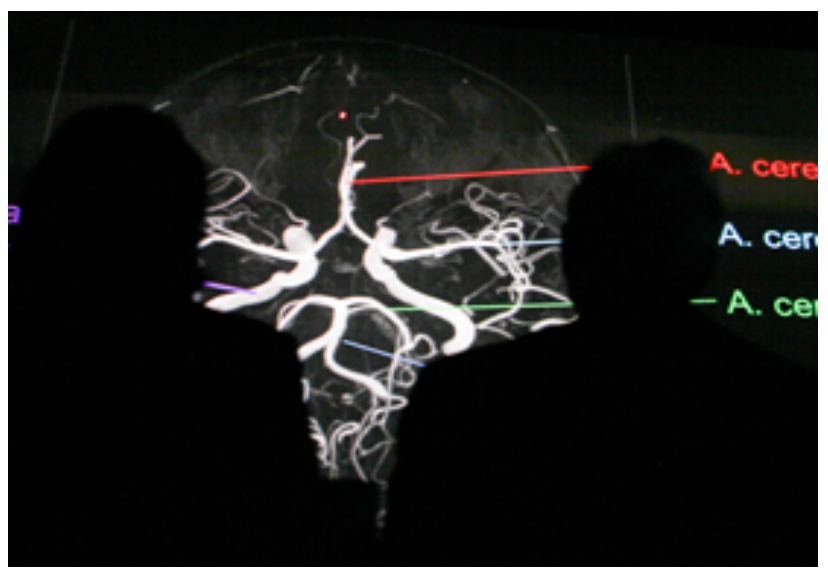

Figure 9: Time-of-flight MR angiography of intracranial vessels; the major arteries are indicated with lines and labeled.

[7] concludes that stereoscopic viewing of images should be further investigated and may well eventually find a permanent place in procedural and diagnostic medical imaging.

In the future, we plan to evaluate different pathological findings in the different body areas, whether these can be visualized sufficiently with the stereoscopic method in the environment of the AEC Deep Space with a projection area of $16 \times 9 \mathrm{~m}$ that we have introduced in this paper. For this purpose, different domain experts have to be involved (e.g. trauma surgeons, abdominal surgeons, neurosurgeons, etc.).

\section{Conclusion}

Ever since this project's inception, the presentation of medical and scientific subject matter to the general public in Deep Space at the AEC has been so successful that the producers have been motivated by popular demand to successively increase the frequency of these events. The highlight is the opportunity to behold CT and MR examinations in high stereoscopic quality on a $16 \times 9$-meter projection surface.

\section{References}

1. Mollberg NM, Parsad NM, Armato SG, Vigneswaran J, Kindler HL, et al (2012) Three-dimensional stereoscopic volume rendering of malignant pleural mesothelioma. Int Surg 97: 65-70. 
Citation: Fellner FA, Berger F, Fellner C, Kremer C, Hörtner H, et al. (2016) Stereoscopic Volume Rendering of Medical Imaging Data for the General Public. J Health Med Informat 7: 213. doi:10.4172/2157-7420.1000213

2. Nakabayashi H, Shimizu K (2012) Stereoscopic virtual realistic surgical simulation in intracranial aneurysms. Neurol India 60: 191-197.

3. Ferroli P, Tringali G, Acerbi F, Aquino D, Franzini A, et al. (2010) Brain surgery in a stereoscopic virtual reality environment: a single institution's experience with 100 cases. Neurosurgery $67: 79-84$

4. Hernes TA, Ommedal S, Lie T, Lindseth F, Lango T, et al. (2003) Stereoscopic navigation-controlled display of preoperative MRI and intraoperative 3D ultrasound in planning and guidance of neurosurgery: new technology for minimally invasive image-guided surgery approaches. Minim Invasive Neurosurg 46: 129-137.
5. Wang W, Lin J, Knosp E, Zhao Y, Xiu D, et al. (2015) Application of MSCTA combined with VRT in the operation of cervical dumbbell tumors. Int J Clin Exp Med 8: 14140-14149.

6. Song SW, Jun BC, Chae SR, Kim BG (2013) Clinical utility of three-dimensional facial computed tomography in the treatment of nasal bone fractures: a new modality involving an air-bone view with a volume rendering technique. Indian J Otolaryngol Head Neck Surg 65: 210-215

7. Stewart N, Lock G, Hopcraft A, Kanesarajah J, Coucher J (2014) Stereoscopy in diagnostic radiology and procedure planning: Does stereoscopic assessment of volume-rendered CT angiograms lead to more accurate characterisation of cerebral aneurysms compared with traditional monoscopic viewing? J Med Imaging Radiat Oncol 56: 172-182. 